\title{
Advanced Nanoscale Characterization of Cement Based Materials Using X-Ray Synchrotron Radiation: A Review
}

\author{
Sejung R. Chae ${ }^{1)}$, Juhyuk Moon ${ }^{1)}$, Seyoon Yoon ${ }^{1)}$, Sungchul Bae ${ }^{1)}$, Pierre Levitz ${ }^{2)}$, \\ Robert Winarski ${ }^{3)}$, and Paulo J. M. Monteiro ${ }^{1), *}$
}

(Received November 30, 2012, Accepted March 18, 2013)

\begin{abstract}
We report various synchrotron radiation laboratory based techniques used to characterize cement based materials in nanometer scale. High resolution X-ray transmission imaging combined with a rotational axis allows for rendering of samples in three dimensions revealing volumetric details. Scanning transmission X-ray microscope combines high spatial resolution imaging with high spectral resolution of the incident beam to reveal X-ray absorption near edge structure variations in the material nanostructure. Microdiffraction scans the surface of a sample to map its high order reflection or crystallographic variations with a micron-sized incident beam. High pressure X-ray diffraction measures compressibility of pure phase materials. Unique results of studies using the above tools are discussed - a study of pores, connectivity, and morphology of a 2,000 year old concrete using nanotomography; detection of localized and varying silicate chain depolymerization in Al-substituted tobermorite, and quantification of monosulfate distribution in tricalcium aluminate hydration using scanning transmission X-ray microscopy; detection and mapping of hydration products in high volume fly ash paste using microdiffraction; and determination of mechanical properties of various AFm phases using high pressure X-ray diffraction.
\end{abstract}

Keywords: X-ray, microscopy, tomography, STXM, X-ray diffraction, high pressure, tobermorite, CSH, fly ash.

\section{Introduction}

The current frontier of cement and concrete research calls for environmental consciousness (Jones et al. 2003). Proposed directions include enhancing the durability characteristics of concrete and therefore extending their serviceable life through modification of existing materials, and developing new blends and materials with effectively low $\mathrm{CO}_{2}$ footprint, such as high volume fly ash mixes or geopolymers and other alternative cementitious binders (Duxson et al. 2007; Juenger et al. 2011). In either case, a careful analysis in the sub micrometer and nanometer scales help to characterize and understand the material in detail.

We present advanced techniques that are innovative not only for their potentials, but also for the benefit of synergistic effects brought on by multi-disciplinary collaborators - this so

\footnotetext{
${ }^{1)}$ Department of Civil and Environmental Engineering, University of California at Berkeley, Berkeley, CA 94720, USA.

*Corresponding Author;

E-mail: monteiro@ce.berkeley.edu

${ }^{2)}$ Laboratory PECSA, CNRS, Université Pierre et Marie Curie, 75252 Paris, France.

${ }^{3)}$ Center for Nanaoscale Materials, Argonne National Laboratory, Argonne, IL 60439, USA.

Copyright $($ The Author(s) 2013. This article is published with open access at Springerlink.com
}

called "big science” era revolutionized by Nobel prize laureate Ernest Lawrence (Yarris 2001) is a sentiment researchers should embrace. As experimental facility users, we work with the staff scientists who specialize in the upkeep and enhancement of highly specialized equipment. With on-going collaboration, the scientists continuously optimize, modify, or build new tools that reflect the research needs of their users. As tools evolve to meet our demands, well-established collaboration is rewarded. This is paramount.

Some of these "big science" based tools - namely, soft $\mathrm{X}$-ray microscopy, hard X-ray microtomography, and neutron diffraction-have been reviewed previously (Monteiro et al. 2009). As an extension to this discussion, several additional synchrotron X-ray based techniques are presented in this paper. A comprehensive review on third generation synchrotron radiation laboratories can be found elsewhere (Schlachter 1994; Attwood 2000; Bilderback et al. 2005; Brown et al. 2006). Briefly, a synchrotron laboratory consists of multiple end stations, or beamlines, illuminated with high flux, highly collimated electromagnetic radiation. This has multiple beneficial implications. First is a potential for a bright incident beam, allowing for fast data collection. This is especially important when numerous acquisitions are required for a single data set, and time induced changes to the sample must be kept at a minimum. Second is selectivity - that is, one can be specific and limit the energy variance (yielding high spectral resolution) or the spot size of the incident beam, while still obtaining statistically significant data counts within a reasonable amount of time. Third is a 
high signal-to-noise ratio $(\mathrm{S} / \mathrm{N})$, which allows for detection and analysis of some of the finer and lower intensity features that would be indistinguishable from background noise if standard laboratory X-ray tools are used instead. Fourth is a relatively straight forward sample preparation requirement. As X-ray based characterization acquired data quality are not sensitive to surface textures of their samples, invasive sample preparation procedure such as abrasive grinding, polishing, or sample coating is often not a requirement for data optimization. Therefore, samples are often probed in their un-treated state, and often scanned at ambient pressures and humidity. Therefore, concerns of chemical and structural alterations to the sample induced by sample preparation are kept at a minimum.

There are numerous synchrotron facilities available worldwide. Each tool is unique and customized differently, and therefore often exhibits a different range of capabilities and limitations. The particular beamlines cited in this paper were chosen based on the compatibility between their specific capabilities and our specific study goals. Also in consideration were location specific restraints, that is, working at a lab in proximity is practically favorable. Table 1 is a comprehensive list of operational user facility synchrotron laboratories in North America, Europe, Japan and Korea that also offer beamlines with similar capabilities highlighted in this paper. We discuss the following techniques:

1. Nanoscale computerized tomography $(n C T)$ : we present soft X-ray illuminated examples from transmission X-ray microscope (TXM) beamline HZB-U41/1-TXM at Berliner Elektronenspeicherring-Gesellschaft für Synchrotronstrahlung (BESSY) in Berlin, Germany; and hard X-ray illuminated examples from the hard X-ray nanoprobe beamline (26-ID-C) operated by the Center for Nanoscale Materials and the advanced photon source (APS) at Argonne National Laboratory. nCT is a X-ray microscope with state-of-art optics available (achieving spatial resolution in the order of $10 \mathrm{~nm}$ ), coupled with a rotational stage, which collects tomography data to create a volumetric representation of the sample. $\mathrm{nCT}$ is ideal for exploring the three dimensional network of complex structures non destructively. For some configurations which will be discussed in the proceeding section, it is also possible to test materials in ambient or simulated environmental conditions. Suitable applications include morphology analysis of hydration products, studies of interface in binding agents, and studies of porosity and its effects on mechanical properties.

2. Scanning transmission X-ray microscope (STXM): we present examples from beamlines 5.3.2.1 and 5.3.2.2 at the advanced light source (ALS), Lawrence Berkeley National Laboratory, Berkeley, CA. STXM is a TXM operated in vacuum with an added ability to perform chemical speciation for grain sizes in the order of $10 \mathrm{~nm}$. Essentially, particle identification through morphology can be supplemented with chemical identity and speciation information, and this is especially useful in soft X-ray microscope problems that deal with multiple phases of materials. In addition, detailed analysis of heterogeneities or chemical changes due to modifiers (such as chemical attacks, on-going hydration, or chemical admixtures) can be monitored across the sample nanostructure.

3. $X$-ray microdiffraction $(\mu X R D)$ : we present examples from beamline 12.3.2 at the ALS. $\mu$ XRD can correlate $\mathrm{X}$-ray fluoresce mapped across the surface of the sample in micrometer resolution with X-ray diffraction patterns obtained with a micrometer sized incident beam. The XRD patterns contain information to identify the crystalline phases present, as well as their implicit orientation, grain size, and strains. $\mu \mathrm{XRD}$ is useful for indexing crystalline phases across a sample's microstructure, as well as tracking localized strains or identifying newly formed phases due to chemical attacks.

4. High pressure X-ray diffraction (HPXRD): we present examples from beamline 12.2.2 at the ALS. HPXRD measures mechanical properties of pure phase materials by directly inducing deformation in the crystal structure of the material. The information obtained from HPXRD is useful in many practical and theoretical applications, such as characterizing phases in existing and new building materials, providing essential information for fracture mechanics modeling, and verifying results from molecular dynamics modeling.

Each of the above techniques will be discussed, with particular attention to their applications in advanced research materials research. We will also provide past and on-going studies as examples.

\section{Nanoscale Computerized Tomography (nCT)}

X-ray microscopy allows for high resolution imaging of X-ray transmission, with near $10 \mathrm{~nm}$ spatial resolution achievable today (Rehbein et al. 2009). A comprehensive review on X-ray microscopy can be found in the literature (Attwood 2000; Howells et al. 1985; Schneider et al. 2012). When nanometer scale X-ray microscopy is combined with a rotational stage, computerized tomography (CT) datasets can be collected. CT, invented by Godfrey Hounsfield in 1972, collects a series of X-ray transmission images of the same sample at numerous rotational angles, and mathematically computes the volumetric locations of the features present in the transmission images to build a 3-dimensional voxel representation of the sample that includes its surface and internal features. These are obtained non-destructively, without inducing fracture on the sample or requiring abrasive techniques that would potentially damage or alter the sample during sample preparation. A comprehensive review of the computations can be found elsewhere (Webb et al. 2002).

When considering the quality of the final reconstruction of $\mathrm{nCT}$, it is important to recognize that certain data acquisition 


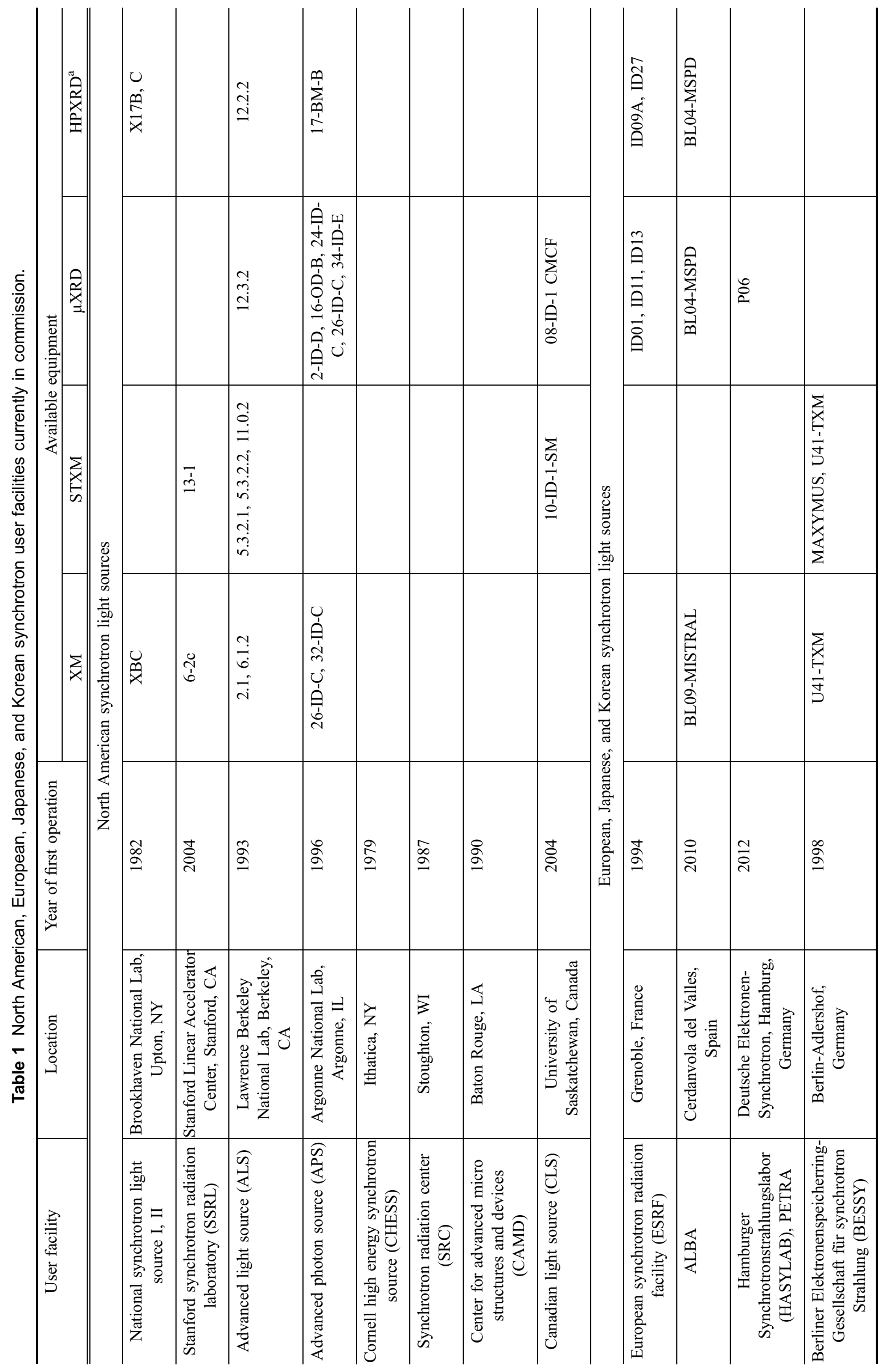

International Journal of Concrete Structures and Materials (Vol.7, No.2, June 2013) | 97 


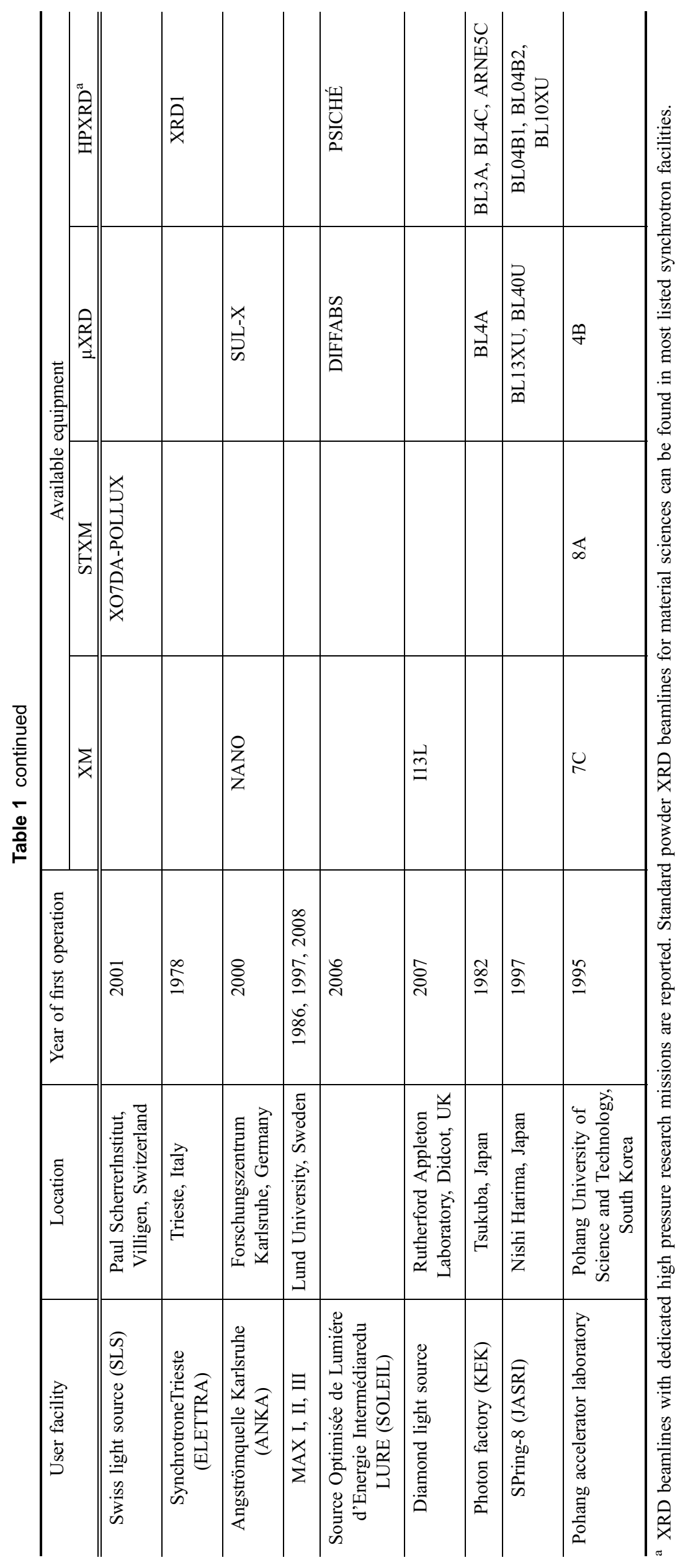

98 | International Journal of Concrete Structures and Materials (Vol.7, No.2, June 2013) 
challenges will induce artifacts, and these artifacts in reconstruction will always compromise the theoretical maximum spatial resolution, that is, the spatial resolution reflected by the raw transmission images. Some of the sources of the artifacts include the following:

Sample size: If the sample size is larger than the field of view (FOV) of the X-ray optical system, then some sample information will be lost invariably and will contribute to reconstruction errors. Therefore, an X-ray optical system with FOV of $15 \mu \mathrm{m}$ and pixel size of $10 \mathrm{~nm}$ will limit the ideal sample size to about $10 \mu \mathrm{m}$ in width in order for the entire sample to fit within the FOV.

In addition, an ideal dataset contains transmission images of partially absorbed X-ray images with sufficient contrast between morphological features. Neither extremely high Xray absorbance nor extremely high $\mathrm{X}$-ray transmission is desirable, as the former is usually coupled with insufficient data collection, and the latter is usually coupled with insufficient contrast between sample features and background. X-ray absorption through matter is expressed by the Beer's law:

$$
I(x)=I_{0} e^{-\mu x}
$$

where $I(x)$ is the final intensity of the beam after the beam passes through sample thickness $x, I_{0}$ is the initial intensity of the beam, and $\mu$ is the material's linear attenuation coefficient, which can be expressed as:

$$
\mu=\sum f_{i} \mu_{i} \rho
$$

where $i$ represents each atomic element, $f_{i}$ is the atomic weight fraction of the element in the material, $\mu_{i}$ is the mass attenuation coefficient at the incident beam energy, and $\rho$ is the density of the material. For example, a $3 \mu \mathrm{m}$ thick calcium hydroxide $\left(\mathrm{Ca}(\mathrm{OH})_{2}\right)$ crystal will exhibit $0.01 \%$ transmission with $500 \mathrm{eV}$ incident beam and $92 \%$ transmission with $8,000 \mathrm{eV}$ incident beam. A sample 10 times thinner, at $0.3 \mu \mathrm{m}$, will exhibit $31 \%$ transmission with $500 \mathrm{eV}$ incident beam and $99 \%$ transmission with $8,000 \mathrm{eV}$ incident beam. This implies that for cementitious materials, as they exhibit fairly comparative $\mu$ as $\mathrm{Ca}(\mathrm{OH})_{2}$, the choice of incident X-ray beam will yield different results and they can be utilized as complementary techniques. In other words, soft X-ray TXM will produce well defined features as long as the sample thickness remains consistently in the order of $0.1 \mu \mathrm{m}$ range. The internal information of the sample, however, is lost when thickness of the sample exceeds the $0.1 \mu \mathrm{m}$ order of magnitude. In contrast, a hard X-ray TXM will yield sufficient transmission for the sample with thickness in the order of $10 \mu \mathrm{m}$. Relative to the soft X-ray TXM, however, the finer sub micron features will be a challenge to distinguish as the transmission contrast is reduced for hard X-rays.

Dimensional and transitional stability: With any tools involved in sub-micron scale imaging, dimensional and transitional stability is essential to produce quality data. While stability of microscope components from vibrations and thermal fluctuations is addressed and optimized in either of the beamlines cited in this section (Rehbein et al. 2009; Robert and Winarski 2012; Schneider et al. 2010), small submicron transitional shifts in between each successive image cannot be avoided. To mitigate these shift errors, gold balls are typically mixed in with the sample, and later used as positional markers to align the transmission images prior to reconstruction. In addition, background normalization is performed to minimize the effects of uneven or shifting illumination intensities.

Finally, sufficient rotational images need to be obtained to produce reconstruction that reflects equal spatial resolution as the transmission images. According to the Nyquist theorem, the minimum number of radiographs needed to achieve this effect within $180^{\circ}$ of rotation is $N \pi / 2$, where $N$ is width of the sample in pixels. In practice, little difference is observed for image numbers $>N \pi / 4$.

Depth of focus (DOF): When DOF is significantly smaller than the FOV of a TXM, the quality of the reconstruction suffers as features farthest from the focal point (FP), and thus extending past the DOF zone, are susceptible to being significantly out of focus. The DOF for a TXM is defined by the following:

$$
D O F= \pm \frac{\lambda}{2 N A^{2}}= \pm \frac{2 \Delta r_{n}^{2}}{\lambda}
$$

where $\lambda$ is the wavelength of the incident X-ray, NA is the numerical aperture of the zone plate, and $\Delta r_{n}$ is the outermost zone width of the objective zone plate. The outermost zone width correlates to the spatial resolution of the TXM image, so we can infer from above equation that decreased spatial resolution and shorter wavelength (harder X-rays) will increase the DOF. For example, for $8 \mathrm{keV} X$-rays the DOF is $14.8 \mathrm{um}( \pm 7.4 \mu \mathrm{m})$ and for $10 \mathrm{keV}$ X-rays the DOF is $18.6 \mathrm{um}( \pm 9.3 \mu \mathrm{m})$. Methods to effectively extend DOF for TXM have been developed (Liu et al. 2012). However, this requires a cumbersome technique of collecting a stack of images taken at multiple sample-to-detector distances, and effectively multiplies total data acquisition time by the number of images collected in each stack set. Therefore, the procedure was not adapted for our studies.

\subsection{Soft X-Ray nCT}

Considering the discussed potential for limitations, an example of soft X-ray TXM and nCT is presented in Fig. 1. The sample is a pure aluminum substituted $11 \AA$ tobermorite phase extracted from an ancient sea water harbor Roman concrete that was cast circa 1st century CE (Jackson et al. 2012). Data was collected from HZB-U41/1-TXM beamline at BESSY. Some limitations in the experimental procedures were imposed. First, the sample was scanned in vacuum, as the absorption of X-rays by the ambient air would be significant considering a $510 \mathrm{eV}$ incident X-ray beam. Second, the sample, in powder form, was dispersed on a custom made copper grid covered with a polyimide membrane $\sim 10 \mathrm{~nm}$ thick. The effective thickness of the film when it was oriented close to parallel to the direction of the beam 
increased sufficiently to significantly absorb the beam, and therefore higher angle rotation data had to be discarded from reconstruction. Essentially, data collection was limited to -75 to $+75^{\circ}$ rotation, collected at $1^{\circ}$ increments and reconstruction algorithm was computed as a partial angle tomography. Avizo software was used to create volumetric reconstruction, which is seen in Fig. 1c.

The reconstruction, with $15.5 \mathrm{~nm}$ voxel length, revealed limited details in the internal structures of the particle which is shown in Fig. 1b. The loss of finer nanostructure detail is evident when compared to a typical TXM image as shown in
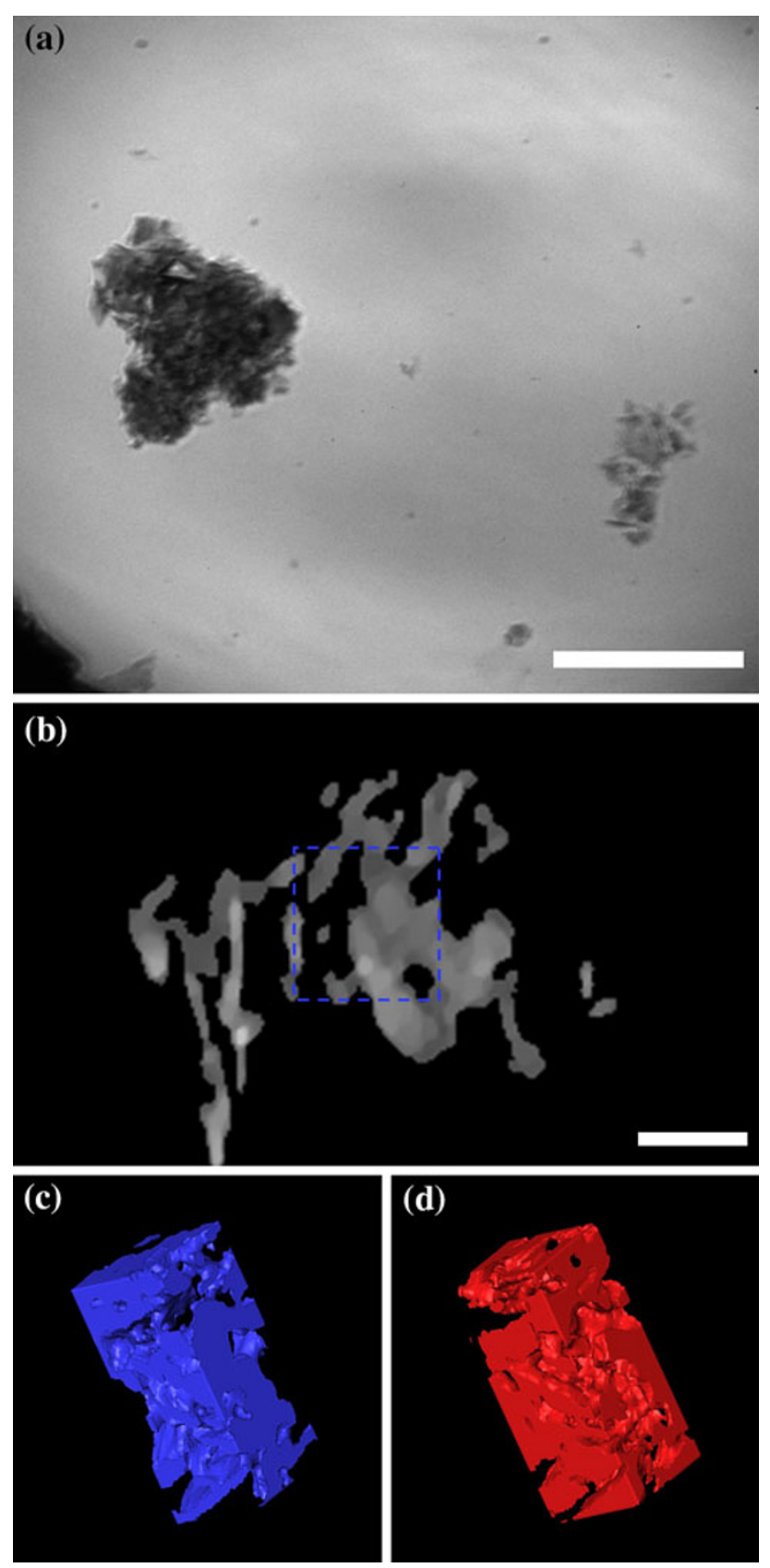

Fig. 1 Soft TXM nCT taken at $510 \mathrm{eV}$ incident X-ray. Typical TXM image is shown in (a), with a $3 \mu \mathrm{m}$ scale bar. A reconstructed tomogram of a particle $\sim 5 \mu \mathrm{m}$ width is shown in (b), with a $1 \mu \mathrm{m}$ scale bar. A 3-D reconstruction of a $1.5 \times 1.5 \times 5 \mu \mathrm{m}$ section (and shown in dotted line in (b)) is shown in (c). Its negative imprint, or the voids, is shown in (d).
Fig. 1a. This information loss can be contributed to three major factors. First is the incomplete set of projections. With $30^{\circ}$ of rotation information missing from the projections, some sample information is lost. The computation challenges associated with partial angle tomography is discussed elsewhere (Schneider et al. 2010). In addition, the large angle increment would also affect the final spatial resolution of the reconstruction. Second is the weak penetration depth of a $510 \mathrm{eV}$ through the sample thickness. Although the existing porosities would indicate that the effective depth of $\mathrm{X}$-ray penetration is smaller than the measured dimensions of the sample, computed attenuated length is $0.44 \mu \mathrm{m}$. As a side note, attenuated length is a computed value using Beer's law, and it is used to indicate optimal transmission image contrast, and it is defined as the total penetration depth at which X-ray transmission is equal to $1 / \mathrm{e}$, or $36.8 \%$. From the tomogram of the sample Fig. $1 \mathrm{~b}$, it is easy to see detect many instances when the X-ray penetration exceeds the attenuated length, which would contribute to a loss of information in the reconstruction. Third is the limited depth of focus. For this particular setting of the optics, the calculated DOF is $2.6 \mu \mathrm{m}$ for a $510 \mathrm{eV}$ incident beam and $40 \mathrm{~nm}$ outer zone width. The DOF is less than the thickness of the sample, and additionally insufficient considering that mechanical limitations of the equipment cause the axis of rotation to shift few microns during the course of its rotation. The deviation from the sample's position away from its ideal FP was evident during data acquisition when portions of the particle were clearly out of focus. This would certainly impose errors during reconstruction.

The reconstruction in Fig. 1c, however, retained excellent topological features, and revealed well-connected solid mass network. In addition, the porosity was rendered (see Fig. 1d, the inverse volume of Fig. 1c). While the voids carry weak quantitative significance when considering the implicit artifacts of the reconstruction, qualitative comparative analysis such as analyzing void morphology and making comparisons with other samples collected with compatible experimental procedures are possible.

Indeed, soft X-ray nCT has proven to be useful for topological reconstruction. In addition, the TXM images obtained in the process contain vivid and contrasting features, and these can be submitted for additional quantitative morphological analysis. This has been demonstrated in a different study performed at the same beamline (Brisard et al. 2012).

\subsection{Hard X-Ray nCT}

With hard X-rays as incident radiation, many of the limitations imposed by soft X-ray nCT are resolved. First, the attenuation length of a carbon-based sample holder is significantly increased, and a full $180^{\circ}$ rotation is possible. Second, the DOF is significantly increased (typically, using the zone plate available, the DOF is about $15-20 \mu \mathrm{m}$ for hard X-ray TXM, as opposed to a several $0.1 \mu \mathrm{m}$ for a soft X-ray TXM). Third, the incident beam absorption due to ambient air is also negligible, and the samples can be tested in ambient conditions which would be crucial for 
experiments that incorporate environmental condition simulations, or for samples like many of the cementitious hydration products that risk structural alterations in vacuum conditions. However, due to the high penetration depth of hard X-rays, reduced absorption contrast is significant. The computed attenuation length of the sample for a $10 \mathrm{keV}$ incident beam exceeds $100 \mu \mathrm{m}$. While X-ray absorption is no longer an issue for hard X-ray illuminated TXM set up, the opposite problem of high radio transparency becomes a source of concern. Often, data interpretation relies heavily on monitoring fringe effects and texture variations that exist in between phases, rather than observing pure absorption contrast.

An example of hard X-ray nCT is shown in Fig. 2. Using the same type of sample from Fig. 1, a tomography dataset was collected from another $11 \AA$ Al-tobermorite particle extracted from ancient Roman sea water harbor concrete, $\sim 9 \mu \mathrm{m}$ thick. Data was collected at the hard X-ray nanoprobe beamline (26-ID-C) operated by the Center for Nanoscale Materials and the APS at Argonne National Laboratory. The sample was mounted on a $10 \mu \mathrm{m}$ thick Kapton tip, and was illuminated with a $10 \mathrm{keV}$ incident beam, with magnification at the detector set at $15.77 \mathrm{~nm}$ pixel size. A total of 1801 images were collected at $0.1^{\circ}$ rotation increment. Spatial resolution was $\sim 30 \mathrm{~nm}$. The DOF at this setting was $\sim 19 \mu \mathrm{m}$. Preliminary background normalization, image alignment, and reconstruction were performed using the XMreconstructor software package from Xradia. A sample transmission image and subsequent reconstruction images are shown in Fig. 2. Compared to the results from soft X-ray nCT, the tomogram in Fig. 2b reveals greater details in inner particle features, although at a significantly reduced contrast relative to the background. The resulting 3-D volume reveals surface topology that is not as vivid as soft $\mathrm{X}$-ray $\mathrm{nCT}$, although it exposes greater details in the internal nanostructure network. The overall quality of the initial reconstruction suggests that further graphic manipulation is needed to clarify the data.

\subsection{Discussion}

As demonstrated, nCT effectively explores nanostructures non destructively. This is useful for characterizing nanoscale topologies, morphology, and to study the porosity network. Concrete, for example, is comprised of a highly heterogeneous mixture of phases with pore diameters in sub-nanometer to centimeter sizes, and these pores influence a multitude of material properties from bulk material strength to durability (Mehta et al. 2006). nCT provides material insights in the sub-micron scale. The ability to scan hard $\mathrm{X}$-ray nCT in ambient conditions allows samples to remain true to their natural state. Dehydration of samples induced by vacuum often raises concerns of misrepresentation as water plays an integral role in the nanostructure of cement hydrated phases.

Additional studies have been conducted to study morphologies of cement hydration products, and to study the interface between the un-reacted raw material and the binding agents of various building materials. Findings based
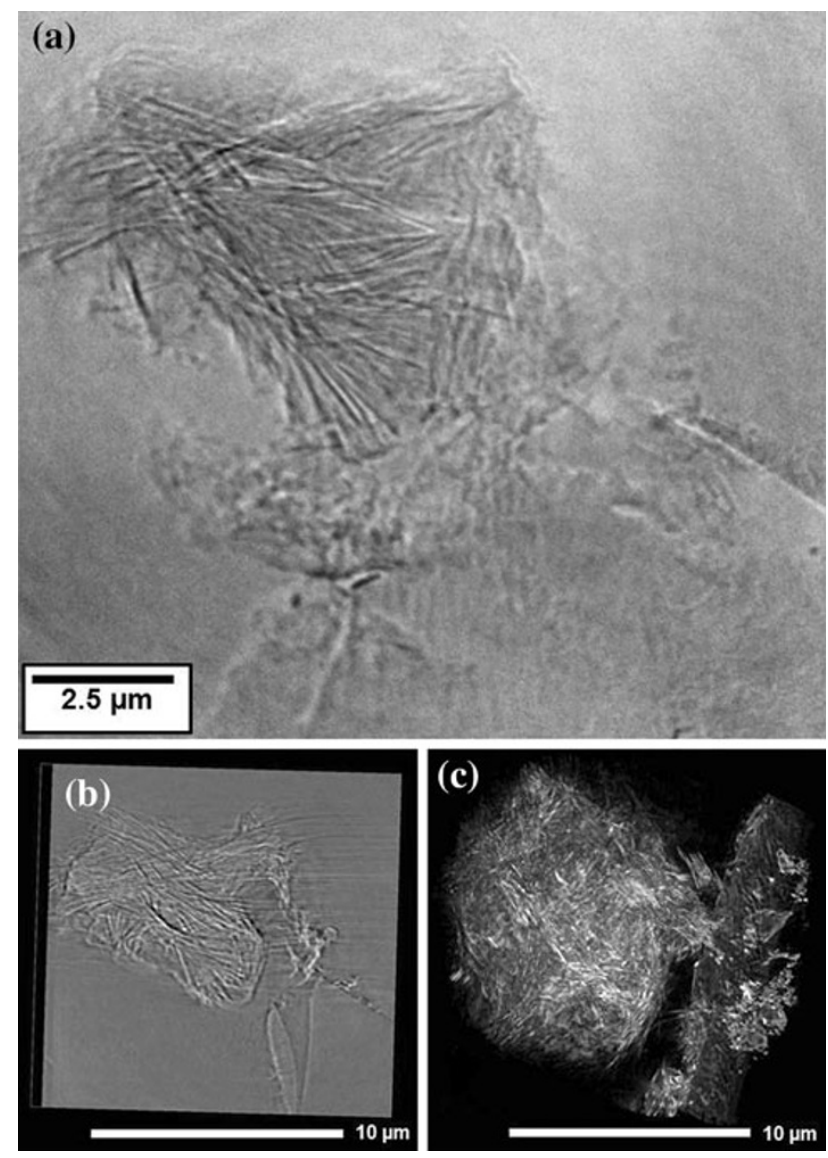

Fig. 2 Hard TXM nCT taken at $10 \mathrm{keV}$ incident beam energy, with a background normalized transmission image, b reconstructed tomogram, and $\mathbf{c}$ the $3-\mathrm{D}$ reconstruction.

on nCT include indexing pore size and connectivity, measuring grain size of crystal phases, making comparisons of implicit binding strength, characterizing material surface texture, observing inter-phase interactions, analyzing inner and surface morphology, and providing models for finite element analysis of the material nanostructure.

\section{Scanning Transmission X-Ray Microscopy}

A wide variety of bulk characterization techniques have been applied to the field of cement and concrete research. One available absorption based technique, which is an efficient method to obtain structural information of cement phases, is nuclear magnetic resonance (NMR) (Jones et al. 2003; Cong and Kirkpatrick 1996). Another technique, Rietveld analysis, allows quantitative phase analysis of multiphase mixtures, and is useful for cement phase analysis (Scrivener et al. 2004). Even though these techniques are able to provide averaged structural or compositional information of cement phases, they cannot be used to understand localized structural changes or spatial distribution of cement phases. Over the past decade, X-ray absorption spectroscopy (XAS) has been developed to improve our understanding of complex natural and engineered materials (Koningsberger and Prins 1987). STXM is one of these techniques. Since it provides spatially-resolved X-ray absorption near edge 
structure (XANES) spectra, STXM/XANES can have a major impact exploring the structure and the composition of cement phases and their hydration process coupled with spatial information. In this section, we review the fundamental aspects of STXM/XANES, and also introduce prospective applications to cement research.

\subsection{STXM/XANES}

Scanning transmission X-ray microscopy provides information on both morphology and XAS. A typical optical arrangement of STXM is shown in 3. The zone plate focuses the incident X-ray photons on the sample. Since the incident $\mathrm{X}$-rays includes significant levels of higher-order diffracted light, the order-sorting aperture (OSA) is used to filter higher-order diffracted light, leaving only the first-order diffracted light. As a set of precise fine stages move the sample to the proper position, the focused X-ray is raster scanned across a selected area of the sample to produce an $\mathrm{X}$-ray microscopic image at a given photon energy. STXM is generally capable of achieving up to $30 \mathrm{~nm}$ spatial resolution. A detailed description of STXM beamlines at the ALS are found elsewhere (Kilcoyne et al. 2003, 2010) (Fig. 3).

In addition, a sequence of images with a predefined energy range and step size can be collected as a "stack." A stack acts as a source to extract XAS data, and a demonstration of how XAS is extracted from a small portion of the image is shown in Fig. 4. Oscillations observed near the absorption edge of XAS form X-ray absorption fine structure (XAFS), which is subdivided into two regions: (1) X-ray absorption near edge structure (XANES), also known as near edge $\mathrm{X}$-ray absorption fine structure (NEXAFS), residing in the region between 10 and $50 \mathrm{eV}$ above the absorption edge; (2) extended X-ray absorption fine structure (EXAFS), in the region up to $1,000 \mathrm{eV}$ above the absorption edge. These regions are illustrated in Fig. 5. In STXM, each aggregate normalized attenuated transmission intensity from a specified area form a single data point representing a single energy value in a XANES plot.

The incident monochromatic X-ray beam excites a core electron of the illuminated atom to an unoccupied bound or to continuum states. This excitation absorbs the X-ray photons, and manifests itself with a step-like absorption edge in the XANES spectra. As shown in Fig. 5, a XANES spectra contains a pre-edge region at energies below the absorption edge, and a post-edge region at energies above

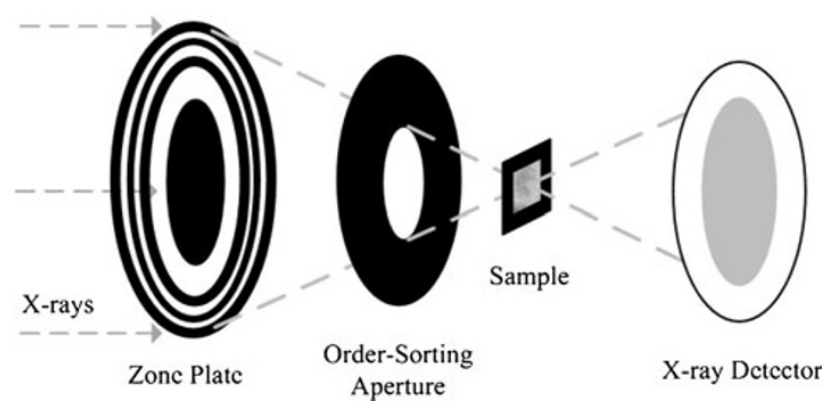

Fig. 3 Schematic representation of the optical elements used in the STXM set-up. the absorption edge. The features in the pre-edge region are usually caused by electron transitions from the core level to unoccupied or partially occupied levels, and require the lowest energy to excite the core electrons. Therefore, the symmetry of the surrounding bonds influences these preedge features. The post-edge features are due to multiple scattering of the excited electrons across neighboring atoms, sometimes extending beyond the first neighboring shell of the bonds. Therefore, these transitions are not localized (Hawthorne 1988). Since the scattering on the neighboring atoms is strong, the excited electrons act as sensitive probes, and we are able to measure the charge distribution and the arrangement of the neighboring atoms surrounding the excited atom.

\subsection{STXM Applications to Cement Research}

Ha et al. (2011) applied STXM and XANES to understand the effects of organic polymers on structures of the calcium silicate hydrate $(\mathrm{C}-\mathrm{S}-\mathrm{H})$ phase. Their research revealed that Si K-edge XANES spectra of STXM images can serve as fingerprints of local structural variations in the tetrahedral silicate chains of the $\mathrm{C}-\mathrm{S}-\mathrm{H}$ phase. Si K-edge generally peaks at a higher energy in its absorption edge with an increase in the silicate tetrahedra polymerization. This study demonstrated that STXM can resolve both the spatial resolution and speciation limitation problems that an NMR analysis would impose. Likewise, Fig. 6b reveals Si K-edge peak shift within different areas of Al-substituted tobermorite which is marked in Fig. 6a. Area 1 has lower binding energy than area 2, suggesting that the silicate chain in area 1 is more depolymerized. In Fig. 7, a molecular model of tobermorite is shown, which is structurally similar to $\mathrm{C}-\mathrm{S}-\mathrm{H}$. Tobermorite is comprised of a $\mathrm{CaO}$ sheet sandwiched between silicate chains, and intermittent absent $\mathrm{SiO}_{4}$ tetrahedra creates a depolymerized silicate chain network. A possible way to explain varying peak positions in Si K-edge XANES is an aggregate variation in these silicate chain lengths at the two localized regions in Fig. 6a.

The $\mathrm{C}-\mathrm{S}-\mathrm{H}$ phase is not only the most abundant hydration product, occupying 50-60\% of the hydrated cement, but it is also the major binding phase of the cement paste, most directly responsible for its engineering properties. This is not because $\mathrm{C}-\mathrm{S}-\mathrm{H}$ is an intrinsically strong phase, but it has a continuous layered structure with cohesive energy to bind particles together. Since the ability of the $\mathrm{C}-\mathrm{S}-\mathrm{H}$ as a binding phase arises from its nanostructure, its internal pore structure and layered structure are worthy of investigation. Therefore, STXM is a promising technique to further understand the structure and the properties of the $\mathrm{C}-\mathrm{S}-\mathrm{H}$ phase. STXM was also used to observe a strong spatial correlation between $\mathrm{Ca}$ and $\mathrm{C}$ XANES spectra, identifying the adsorbed sites of organic polymers in the interlayer of the $\mathrm{C}-\mathrm{S}-\mathrm{H}$ phase $(\mathrm{Ha}$ et al. 2011). Further studies are being performed to study the interaction between cementitious materials and organic admixtures such as super plasticizer, air-entraining admixtures, and shrinkage reducing admixtures.

In addition to their structural information, XANES spectra can be used to quantify phase compositions from reference 

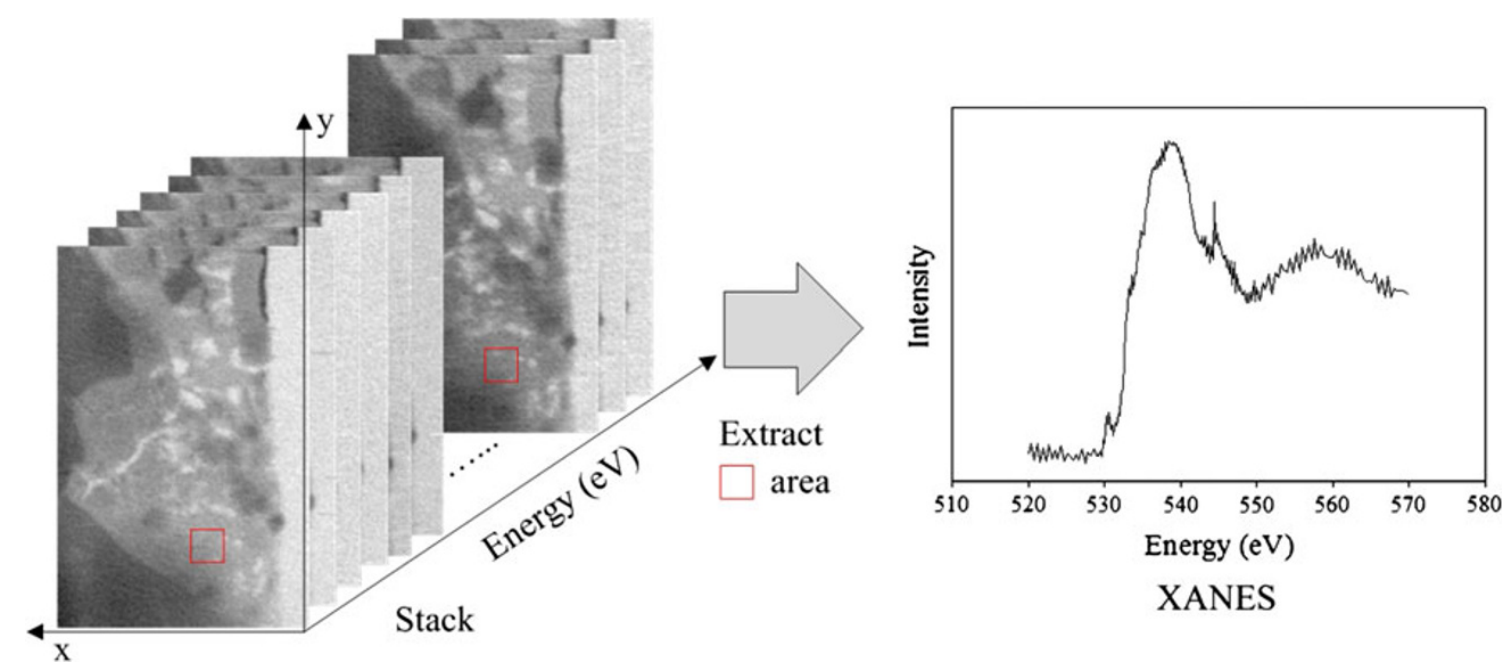

Fig. 4 XANES extraction from the stack, a collection of single images at incremental energy levels.

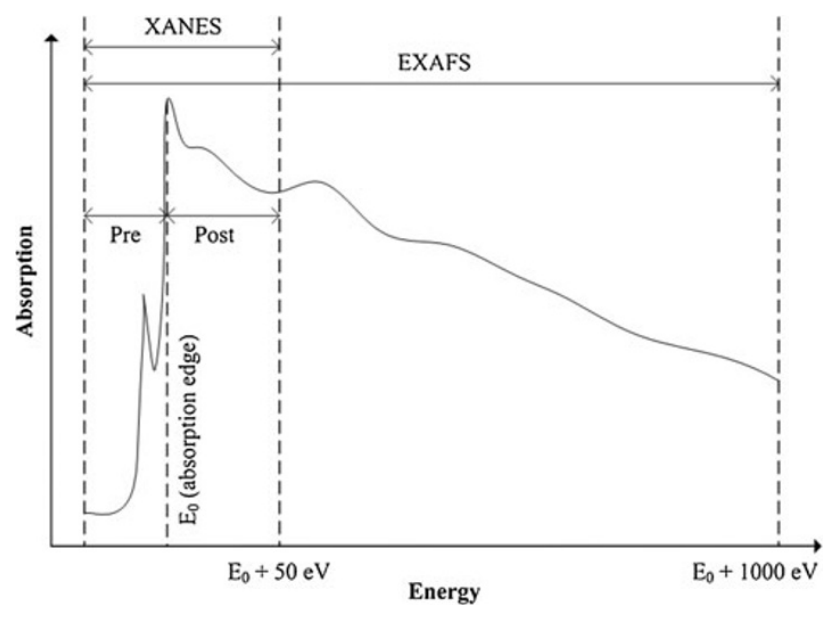

Fig. 5 XAS example depicting the XANES and EXAFS regions as well as the pre and post absorption edge regions.

spectra to derive quantitative composition maps. Wieland et al. (2010) applied XANES spectra to quantify Al and S-bearing mineral in the hardened cement paste. The leastsquare linear combination (LC) fitting was performed to determine the contribution of reference compounds to experimental XANES spectra (Bentz 1997). Figure 8a shows the LC fitting of a $\mathrm{Ca}_{\mathrm{III}, \mathrm{II}}$-edge XANES obtained from $\mathrm{C} 3 \mathrm{~A}$ hydration, and Fig. $6 \mathrm{~b}$ shows the spatial distribution of monosulfate over hydrated $\mathrm{C} 3 \mathrm{~A}$ paste computed with LC fitting. STXM/XANES offer a great potential for investigating complex reactions and highly heterogeneous samples such as cement because it produces spatiallyresolved molecular-level information.

\section{X-Ray Microdiffraction}

Since von Laue in 1912 and Braggs in 1913 discovered that crystalline substances act as diffraction gratings for X-ray wavelengths similar to the spacing of planes in a crystal lattice, XRD has been utilized to investigate the long range periodicity of atomic structures in crystals. XRD is a
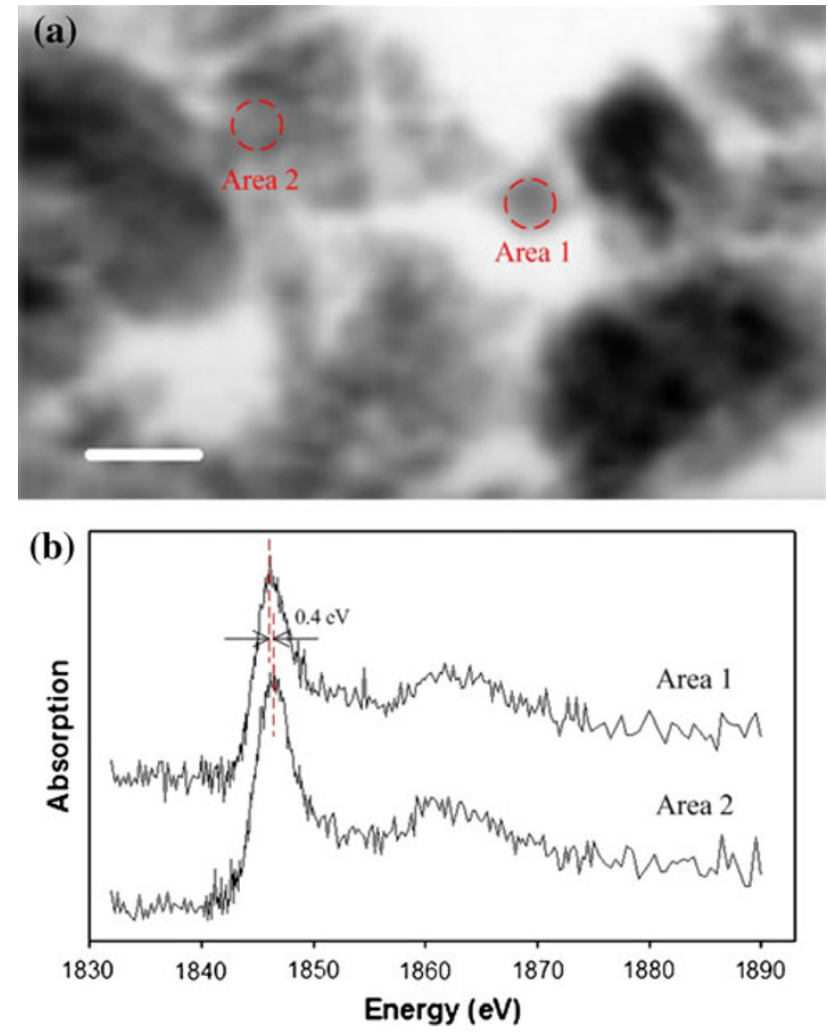

Fig. 6 a STXM image of the Al-substituted C-S-H phase: the scale bar is $1 \mu \mathrm{m}$. b Si K-edge XANES spectra of the corresponding areas in (a).

very versatile tool and its data provide us with information on the phase identification of an unknown crystalline material; the atomic spacing; the orientation of a single crystal or grain; and the size, shape and internal stress of small crystalline regions (Cullity 1978; Langford 1975). Compared to standard lab-based X-ray powder diffractometer, $\mu \mathrm{XRD}$ is a structural analysis tool which allows for the examination of the samples in micron- or sub-micron scale and yields greater precision in lattice parameter determination. It is often used to complement X-ray microspectroscopy including X-ray microfluourescence, extended EXAFS, and NEXAFS. This advanced technique has been widely adapted to investigate the mesoscale plastic deformation 


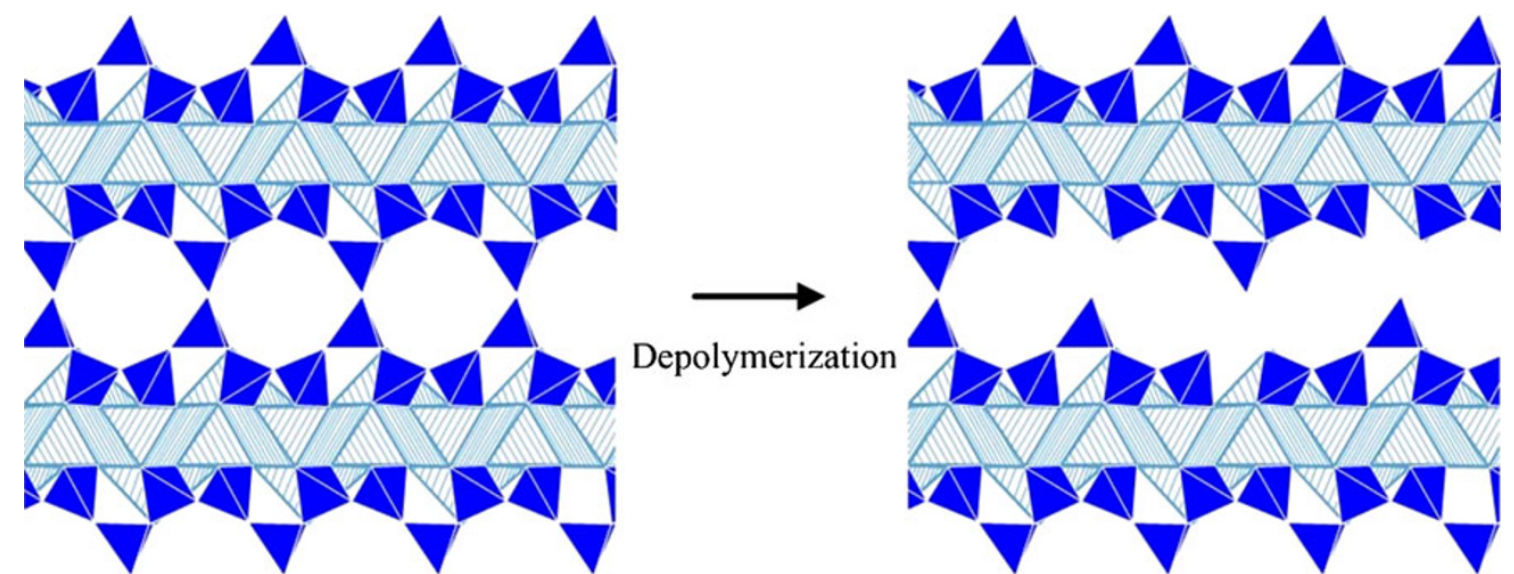

Fig. 7 Illustration of depolymerization of the silicate chains in tobermorite structure.

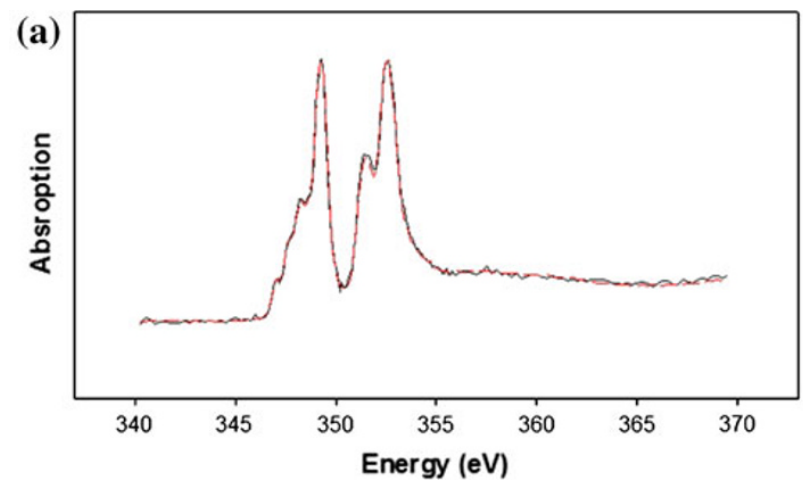

(b)

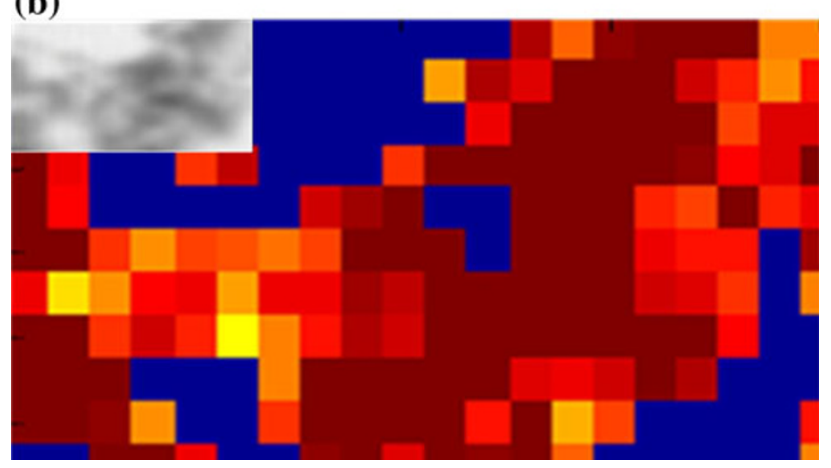

Fig. 8 a XANES spectrum of $\mathrm{Ca} \mathrm{L}_{\mathrm{III}, \mathrm{II}}$-edge obtained from the C3A hydration product: solid lines are the experimental XANES spectrum, and the dashed line is the modeled spectrum based on LC fitting b Contour map of the C3A hydration product showing the monosulfate distribution based on the LC fitting result.

mechanisms in individual grains within a polycrystalline thin film (Nyilas et al. 2009), microstructure of cerium oxide plastic (John 2003), elastic strains in cracks in alloy (Chao et al. 2012), and it also implemented for applications in the field of biology and archeology (Cedola et al. 2004; Gilbert et al. 2008).

Synchrotron radiation provides ultra-bright ultraviolet light and X-rays which are used for a wide range of analytical techniques. Beamlines that offer X-ray beam size at micron or sub-micron dimensions are available at several synchrotron facilities including the ALS, APS, European synchrotron radiation facility (ESRF), National synchrotron light source (NSLS), Canadian light source (CLS), Swiss light source (SLS) and Pohang light source (PLS). Among those beamlines, beamline 12.3.2 at the ALS is a versatile $\mathrm{X}$-ray microdiffraction station recently built on a superbend magnet source to deliver an X-ray spectrum ranging from 5 to $22 \mathrm{keV}$ with high photon flux and rapid data collection capabilities (Tamura et al. 2009; Kunz et al. 2009).

Schematics of $\mu$ XRD at beamline 12.3.2 at the ALS is described by Tamura et al. (2009). Briefly, the hard X-ray beam is focused down to $\sim 1 \mu \mathrm{m}$ size at the sample position using a pair of elliptically bent Kirkpatric-Baez (KB) mirrors enclosed in a vacuum box. KB mirror improves the resolution into the nanometer scale. The sample placed on high precision stages can be raster-scanned under the microbeam while a diffraction pattern is taken at each step. Both polychromatic and monochromatic radiation modes can be conducted by $\mu \mathrm{XRD}$. Polychromatic beam is best suited for polycrystalline sample with grain size larger than the beam size, while monochromatic beam is best suited for polycrystalline sample with grain size smaller than the beam size. Typically, monochromatic beam mode is suitable for cementitious material, which is polycrystalline and heterogeneous. Laue diffraction pattern (single crystal pattern) is constructed when polychromatic beam is used, and Debye-Scherrer diffraction pattern (powder ring pattern) is constructed when monochromatic beam is used. Examples of these patterns are presented in Figs. 9a and 9b.

In addition, $\mu \mathrm{XRD}$ operates both in reflection and transmission modes. Typically, thin-sectioned samples are used for the measurement in transmission mode. Each diffracted pattern can be used to index the crystalline phases in the sample. Furthermore, the arrays of diffraction patterns collected by a MAR133 $\mathrm{X}$-ray detector are analyzed to derive the distribution maps of phases. Stress, strain and plastic deformation within the sample can also be mapped (Rehbein et al. 2009). Further details of the instrument are explained by Tamura et al. (2009). Data are analyzed with X-ray microdiffraction analysis software (XMAS), which is used to extract quantitative information from polychromatic and monochromatic beam patterns. With XMAS, thousands of patterns can be indexed in one session, and these patterns are converted into 2-D element mapping by individual diffraction patterns (Tamura et al. 2003).

$\mathrm{X}$-ray microdiffraction is an effective tool with applicable methods to study a wide range of scientific fields. We have 

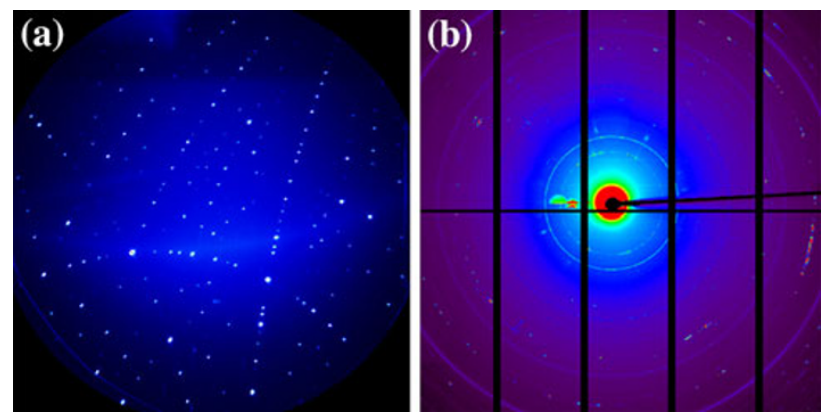

Fig. 9 a Laue diffraction pattern of a crystalline phase found in type $\mathrm{F}$ fly ash obtained with polychromatic (white) beam radiation b Debye-Scherrer diffraction pattern from a HVFA paste microstructure obtained with monochromatic beam radiation.

applied $\mu$ XRD technique to study the hydration products of cementitious material based systems and corrosion of reinforcing steel bars in concrete. In a previous study, we were able to quantify the orientation and the distribution of ettringite crystals $\left[\mathrm{Ca}_{6} \mathrm{Al}_{2}(\mathrm{OH})_{12}\left(\mathrm{SO}_{4}\right)_{3}{ }_{2} 26 \mathrm{H}_{2} \mathrm{O}\right]$ which led to the expansion and cracking of hydrated Portland cement system through sulfate attack (Wenk et al. 2009). Diffraction images were analyzed using Rietveld method to obtain information on crystal textures. It was found that the $\mathrm{c}$ axis of the trigonal ettringite crystal, which is also the elastically stiffest direction, was preferentially oriented perpendicular to the fracture surfaces.

We are also studying the characteristics of $\mathrm{C}-\mathrm{S}-\mathrm{H}$ gels, undoubtedly the most important phase in cementitious material based systems, in high volume fly ash (HVFA) paste. Thin sectioned $(\sim 200 \mu \mathrm{m})$ HVFA paste was used and the experiment was conducted in transmission mode. Compared with the results from standard lab-based powder XRD, synchrotron $\mu$ XRD method has proven to be much more effective in identifying minute amounts of phases localized in small areas of the heterogeneous HVFA paste. Furthermore, we were able to differentiate the properties of $\mathrm{C}-\mathrm{S}-\mathrm{H}$ in HVFA paste from pure Portland cement paste. In addition, obtained results allow a better visualization of the microstructure of the HVFA paste with contributing information from the X-ray microfluorescence ( $\mu \mathrm{XRF}$ ) maps. This provides a deeper understanding of various hydration product formations. An example of an unindexed diffraction pattern from HVFA paste microstructure is shown in Fig. 9b.

In addition to studying the hydration products in cement based systems, we also investigated corrosion products formed on corrosion resistant reinforcing steel in concrete exposed to sea water. We are able to differentiate various crystalline phases in corrosion products, and we were able to determine their distribution in steel-concrete interface with qualitative chemical analysis through $\mu \mathrm{XRF}$ mapping. Detailed experimental results of aforementioned studies will be reported in future publications.

\section{High-Pressure X-Ray Diffraction}

Mechanical performance of building materials is crucial for its use. Although we can easily conduct a macro-scale compressive test on concrete, nano-mechanical properties of crystals inside the concrete provide essential information for understanding overall mechanical properties of composites, and help develop high performance concrete. There are several methods to measure the mechanical properties in nano-scale. Nano-indentation technique measures indentation modulus (Constantinides and Ulm 2004), and Brillouin spectroscopy reveals full elastic constants and averaged mechanical properties (Speziale et al. 2008). Although these techniques determine mechanical properties of a material, detailed atomistic information due to deformation remains a mystery. HPXRD experiments help perform detailed structural mechanism. From HPXRD we can derive the mechanical property of the crystals and information of atomistic structural mechanism of the crystals. In addition, phase transition of materials involves discontinuities in the first derivatives of free energy with respect to their volume. From HPXRD, we could measure the pressure-volume behavior of pure materials directly. Since the pressure is a first derivative of free energy with respect to volume, thermodynamic stability of materials could be indirectly estimated by the HPXRD.

In this section, measurement of the mechanical property of AFm phases $\left(\mathrm{Al}_{2} \mathrm{O}_{3}-\mathrm{Fe}_{2} \mathrm{O}_{3}\right.$-mono) will be reviewed (Moon et al. 2011, 2012). They are among the hydration products of Portland cement and responsible for many durability issues in concrete. The main crystal structure of the AFm phases consists of principal layer $\left[\mathrm{Ca}_{2}(\mathrm{Al}, \mathrm{Fe}) \cdot(\mathrm{OH})_{6}\right]^{+}$and interlayer region $\left[\mathrm{X}_{\mathrm{n}} \mathrm{H}_{2} \mathrm{O}\right]^{-}$. The $\mathrm{X}$ anion is hydroxide, sulfate, chloride, or carbonate. Detailed crystal structure of monocarboaluminate $\left(\mathrm{C}_{4} \mathrm{~A} \overline{\mathrm{C}} \mathrm{H}_{11}\right)$ is shown in Fig. 10.

\subsection{Experimental Method}

Pressure-volume data were obtained by powder X-ray diffraction at beamline 12.2.2 at the ALS (Kunz et al. 2005). This beamline benefits from hard X-ray radiation generated by a superbend magnet. The beamline operates in angle dispersive geometry and is equipped with a MAR3450 image plate detector and goniometry for powder diffraction measurements from samples placed in a diamond anvil cell (DAC).

High-pressures were generated using the DAC. Samples are contained between two round cut diamonds with their table facets facing the opposite direction. A thin metal foil is pre-compressed between the two diamonds and a small hole is drilled in the center to create the sample chamber. In most cases, stainless steel is an acceptable material for the foil, but an incompressible rhenium foil is required for experiments involving extremely high pressures. The sample is held together with a few micron-sized chips of ruby, which are used as pressure gauge, and a pressure transmitting medium is loaded into the hole in the gasket. For the pressure transmitting medium, silicone oil (a mixture of polysiloxane chains with methyl and phenyl groups), or methanol and ethanol mixture are popular. The pressure transmitting medium ensures that the sample is pressurized at a hydrostatic pressure. Pressing together and releasing apart the diamonds induces and relaxes the pressure on the sample. 


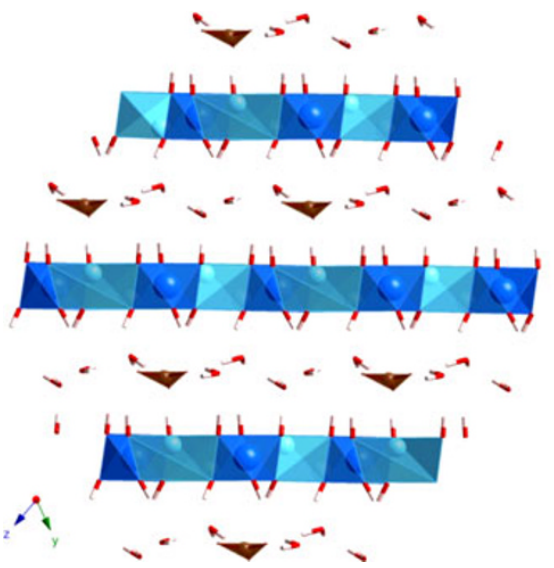

Fig. 10 Crystal structure of monocarboaluminate; (left) general view, and (right) detail of $\mathrm{Ca}-\mathrm{Al}-\mathrm{O}$ interlayer. Dark blue, light blue and brown polyhedra are, respectively,

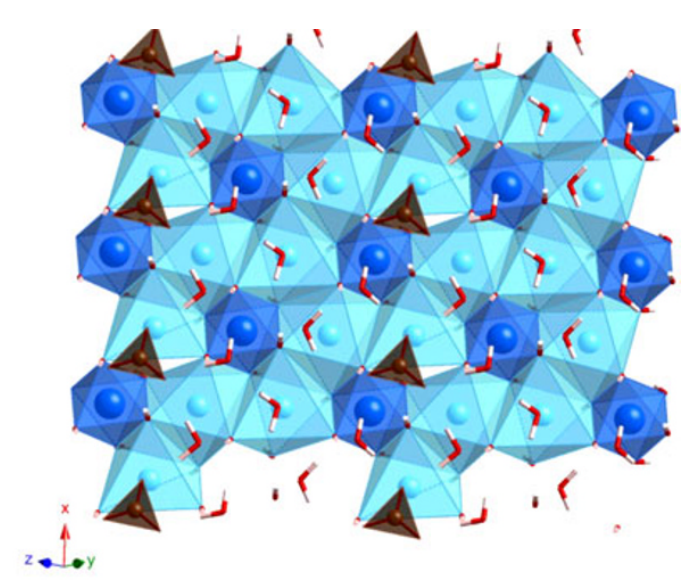

sixfold Al polyhedra, sevenfold Ca polyhedra and carbonate oxide group. White-red bars hydrogenoxygen bonds of hydroxide and water molecules.
Figure 11 shows X-ray patterns of monocarboaluminate at different pressures (Moon et al. 2012). The National Bureau of Standard's LaB6 powder diffraction standard was used to calibrate the sample-to-detector distance, which was computed to be precisely $357.1010 \mathrm{~mm}$. Monochromatic $25 \mathrm{keV}$ hard X-ray was selected as the incident beam. Finally, pressure dependent behavior of the sample was measured from structural refinements at different pressures. As shown in Fig. 11, the diffraction peaks sometimes become diffuse with increasing DAC pressures. Most crystal structures in hydrated cements have interlayer spaces which may contain water and/or various guest molecules. Infiltration of pressure transmitting solution into these interlayer regions, and vibration of atoms under pressure are usually responsible for the diffuse X-ray patterns. Therefore, selecting the appropriate pressure transmitting solution and incident X-ray energy value are crucial for obtaining high-quality data for cementitious materials.

\subsection{Results}

From the obtained $P-V$ data, we can calculate an isothermal bulk modulus of materials. The Birch-Murnaghan equation of state (BM EoS) is used in most cases (Birch 1978),

$$
P=\frac{3}{2} K_{0}\left[\left(\frac{V_{0}}{V}\right)^{\frac{7}{3}}-\left(\frac{V_{0}}{V}\right)^{\frac{5}{3}}\right]\left\{1+\frac{3}{4}\left(K_{0}^{\prime}-4\right)\left[\left(\frac{V_{0}}{V}\right)^{\frac{5}{3}}-1\right]\right\}
$$

where $\mathrm{V}$ is volume of the unit cell, $\mathrm{V}_{0}$ is volume at zero pressure, $\mathrm{P}$ is the pressure applied to the sample, $\mathrm{K}_{0}$ it the isothermal bulk modulus at zero pressure, and $\mathrm{K}_{0}{ }^{\prime}$ is the first derivative of the bulk modulus at zero pressure. The fitting results of various AFm phases are summarized in Table 2.

Figure 12 illustrates pressure dependent behaviors of the AFm phases. In the case of hemicarboaluminate and strätlingite, pressure-induced dehydration occurs at fairly low pressures (Moon et al. 2011). Water molecules in the interlayer migrate away from the crystal structure at a specified pressure, and this behavior is accelerated by the presence of hygroscopic fluid such as the methanol/ethanol mix. This causes a stepwise shrinkage of layer spacing and makes the crystal structure significantly incompressible. This phenomenon was confirmed by comparing the effects of using two different types of pressure-transmitting fluids. Monocarboaluminate exhibits stable behavior when pressurized. Accordingly, the measured isothermal bulk modulus of monocarboaluminate is significantly higher than that of other AFm phases. We can infer from this experiment that the fully incorporated carbon-oxide groups in the monocarboaluminate play a key role in the mechanical performance of the crystal. On the other hand, partially occupied carbonoxide groups of hemicarboaluminate allow more water molecules to reside in the interlayer region, and make the crystal structure mechanically unstable. The strengthening of AFm structure by incorporation of carbon is essential to understanding the mechanical impact of limestone added cement and carbon sequestration in concrete.

\subsection{Discussion}

Recently, mechanical properties of calcium aluminate hydrates as well as calcium silicate hydrates have been successfully tested using HPXRD (Moon et al. 2011, 2012; Oh et al. 2011, 2012; Clark et al. 2008). The application of the technique to cement and concrete research has many potential opportunities. HPXRD is a popular method in geophysics for testing mantle materials under extreme pressure and temperature conditions (Ohuchi et al. 2011). Therefore, various types of DAC with laser heating device have been developed at the HPXRD beamline. Taking advantage of the DACs, thermal expansion coefficient and pressure-volume-temperature diagram of cementitious materials could be obtained (Ohuchi et al. 2011).

Although volume or density of crystalline materials can be measured with XRD techniques, the method for amorphous materials has not yet been fully established. Combining XRD and X-ray absorption was developed and successfully tested on noncrystalline materials (Hong et al. 2007; Sato and Funamori 2008). Instead of measuring volumes, densities of material at different pressures can be obtained from 


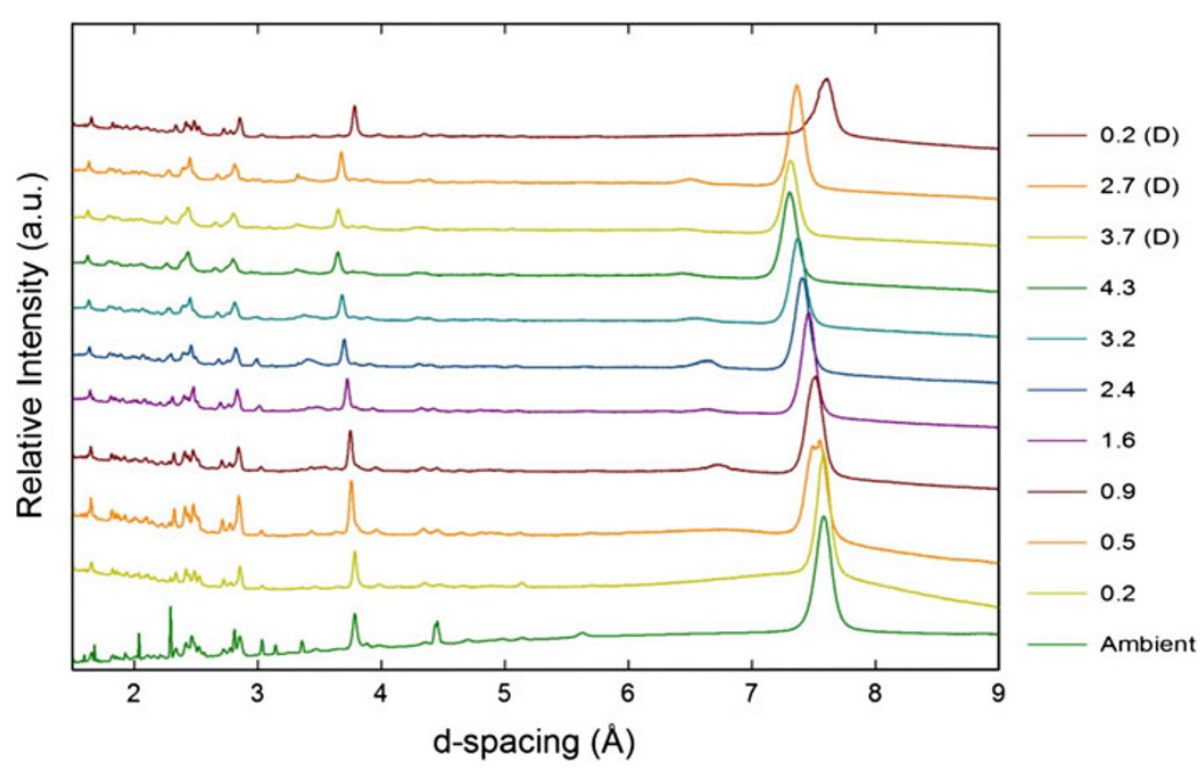

Fig. 11 Integrated X-ray diffraction patterns of monocarboaluminate with different pressures. The numbers indicate applied hydrostatic pressures (GPa). The top three patterns (D) were collected during decompression (Moon et al. 2012).

Table 2 Summary of isothermal bulk modulus and its first derivative of calcium aluminates phases.

\begin{tabular}{|c|c|c|c|c|c|c|c|c|}
\hline & Method & $\begin{array}{c}\text { Pressure range } \\
(\mathrm{GPa})\end{array}$ & \begin{tabular}{|c} 
Initial volume \\
$\left(\AA^{3}\right)$
\end{tabular} & $K_{0}^{\prime}$ & $K_{0}(\mathrm{GPa})$ & Fixed $K_{0}^{\prime}$ & $K_{0}(\mathrm{GPa})$ & \\
\hline $\begin{array}{l}\text { Monocar- } \\
\text { boaluminate } \\
\mathrm{C}_{4} \mathrm{~A} \overline{\mathrm{C}} \mathrm{H}_{11}\end{array}$ & $\begin{array}{c}\text { HPXRD (SO, } \\
x=11)\end{array}$ & $0.1-4.3$ & $433(2)$ & 5.02 & $53(5)$ & 4 & $54(4)$ & $\begin{array}{l}\text { Moon et al. } \\
\text { (2012) }\end{array}$ \\
\hline \multirow{2}{*}{$\begin{array}{l}\text { Hemicar- } \\
\text { boaluminate } \\
\mathrm{C}_{4} \mathrm{~A}_{\bar{C}_{0.5}} \mathrm{H}_{12}\end{array}$} & HPXRD (SO) & $0.1-1.1$ & $1,418.04(1)$ & n.d & n.d & 4 & $15(2)$ & \multirow{3}{*}{$\begin{array}{l}\text { Moon et al. } \\
\text { (2011) }\end{array}$} \\
\hline & HPXRD (ME) & $0.1-1.8$ & $1,418.94(4)$ & 13.6 & $9(2)$ & 4 & $14(1)$ & \\
\hline $\begin{array}{c}\text { Strätlingite } \\
\mathrm{C}_{2} \mathrm{ASH}_{8}\end{array}$ & HPXRD (SO) & $0.1-1.5$ & $1,077.30(2)$ & n.d. & n.d. & 4 & $23(2)$ & \\
\hline $\begin{array}{l}\text { Ettringite } \\
\mathrm{C}_{6} \mathrm{AS}_{3} \mathrm{H}_{32}\end{array}$ & HPXRD (SO) & $0.1-1.2$ & $2,352.8(1)$ & - & - & 4 & $27(7)$ & $\begin{array}{c}\text { Clark et al. } \\
\text { (2008) }\end{array}$ \\
\hline
\end{tabular}

$n d$ not determined, $S O$ silicone oil, $M E$ mixture of methanol and ethanol.

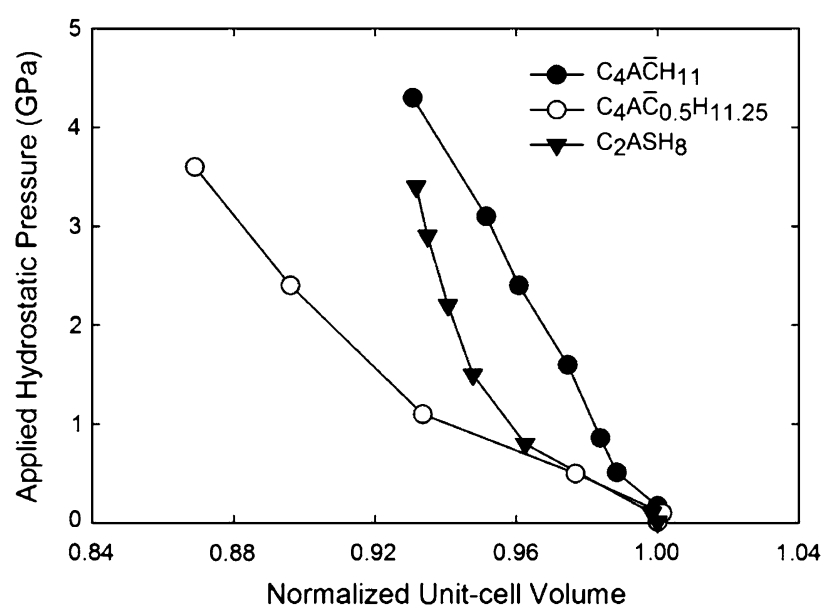

Fig. 12 Normalized unit cell volumes of AFm phases as a function of pressure (Moon et al. 2011, 2012).

using DAC and two reference materials. In addition, pairdistribution function (PDF) measurements can be applied with a wide angle DAC. By fitting structural factors of noncrystalline samples under different pressure conditions, densities of the materials at these pressures can be obtained. However, this method requires background subtraction in order to get the PDF profile of the samples. This is a challenging task in high-pressure experiments because of the inherent difficulty in obtaining pure background patterns (i.e. X-ray scattering profiles of diamonds and gasket without any sample). Only a few amorphous materials have been analyzed using this method (Shen et al. 2002; Xiao et al. 2010; Eggert et al. 2002).

Finally, the compressibility of crystals can be theoretically calculated from density function calculations or molecular dynamic simulations (Moon et al. 2012). Experimentally obtained bulk modulus of cementitious materials will be used to validate the performance of different force-field potentials in molecular dynamic simulations. Precisely designed empirical potentials are important elements to accurately simulate static and time-dependant behavior of materials. HPXRD is one of a few existing experimental techniques to measure the nano-mechanical properties 
directly and to provide detailed atomistic information of materials under various conditions. HPXRD produces valuable data which helps us to understand the behavior of cementitious materials at nano-scale and to support the various atomic level simulations.

\section{Conclusions}

Our experiences with advanced synchrotron based tools have proven to significantly enhance the understanding of our materials. These novel techniques are applicable to a wide range of material science studies, and provide opportunities to extract otherwise unattainable specific information.

In nanotomography, non destructive 3 -D imaging provides volumetric insights into the morphology, bonding, and interactions between various phases and pores in our material. The technique can be applied to new cementitious blends and materials, and successful quantification has been demonstrated.

In scanning transmission X-ray microscopy, chemical speciation via absorption spectroscopy is coupled with spatial information provided by transmission imaging. A heterogeneous sample can be analyzed by tracking localized variations in chemical bonding. With further analysis, quantitative composition analysis is also possible.

Microdiffraction coupled with micro X-ray fluorescence capabilities allows us to combine quantitative element mapping with microscale crystallography variations. This is especially useful in indexing heterogeneous mixes, and for precise mapping of localized crystalline concentrations.

In high pressure $\mathrm{X}$-ray diffraction, deformation is induced directly on the crystal cell of the material to determine its mechanical properties. Analysis has also been performed on amorphous and poorly crystalline materials. Future studies can also incorporate effect of temperature on material properties.

\section{Acknowledgments}

This publication is based on studies supported in part by Award No. KUS-11-004021, made by King Abdullah University of Science and Technology (KAUST) and by National Institute of Standards and Technology (NIST) Grant 60NANB10D014. We thank Helmholtz-Zentrum Berlin (HZB) for the allocation of beamtime at the soft $\mathrm{X}$-ray microscope at Berliner Elektronenspeicherring-Gesellschaft für Synchrotronstrahlung (BESSY); and to Peter Guttmann and Katja Henzler for their scientific support at the HZB-U41/1-TXM beamline. Use of the hard X-ray nanotomography beamline at the Center for Nanoscale Materials was supported by the U. S. Department of Energy, Office of Science, Office of Basic Energy Sciences, under Contract No. DE-AC02-06CH11357. The data for STXM (beamlines 5.3.2.1 and 5.3.2.2), microdiffraction (beamline 12.3.2), and HPXRD (beamline 12.2.2) were acquired at the Advanced Light Source, supported by the Director of the
Office of Science, Department of Energy, under Contract No. DE-AC02-05CH11231. We thank David A. Kilcoyne, Tolek Tyliszczak, Martin Kunz, Nobumichi Tamura, and Simon Clark for their scientific support at the Advanced Light Source. We are grateful for Marie D. Jackson and the Romacons drilling project in collaboration with CTG Italcementi in Bergamo, Italy, for the procurement and preparation of the ancient Roman harbor concrete samples. Finally, we thank Kang Su Kim for his valuable discussions during the production of this paper.

\section{Open Access}

This article is distributed under the terms of the Creative Commons Attribution License which permits any use, distribution, and reproduction in any medium, provided the original author(s) and the source are credited.

\section{References}

Attwood, D. (2000). Soft X-rays and extreme ultraviolet radiation: Principles and applications. USA: Cambridge University Press.

Bentz, D. P. (1997). Three-dimensional computer simulation of Portland cement hydration and microstructure development. Journal of the American Ceramic Society, 80, 3-21.

Bilderback, D. H., Elleaume, P., \& Weckert, E. (2005). Review of third and next generation synchrotron light source. Journal of Physics B, 38, 773-797.

Birch, F. (1978). Finite strain isotherm and velocities for singlecrystal and polycrystalline $\mathrm{NaCl}$ at high pressures and $300^{\circ} \mathrm{K}$. Journal of Geophysical Research, 83, 1257-1268.

Brisard, S., Chae, R. S., Bihannic, I., Michot, L., Guttmann, P., Thieme, J., et al. (2012). Morphological quantification of hierarchical geomaterials by X-ray nano-CT bridges the gap from nano to micro length scales. American Mineralogist, 97, 480-483.

Brown, G. E., Sutton, S. R., \& Calas, G. (2006). User facilities around the world. Elements, 2, 9-14.

Cedola, A., Lagomarsino, S., Komlev, V., Rustichelli, F., Mastrogiacomo, M., Cancedda, R., et al. (2004). High spatial resolution X-ray micro diffraction applied to biomaterial studies and archeometry. Spectrochimica Acta Part $B, 59,1557-1564$.

Chao, J., Suominen Fuller, M. L., Sherry, N., Qin, J., McIntyre, N. S., Ulaganathan, J., et al. (2012). Plastic and elastic strains in short and long cracks in alloy 600 studied by polychromatic X-ray micro diffraction and electron backscatter diffraction. Acta Materialia, 60, 5508-5515.

Clark, S. M., Colas, B., Kunz, M., Speziale, S., \& Monteiro, P. J. M. (2008). Effect of pressure on the crystal structure of ettringite. Cement and Concrete Research, 38, 19-26.

Cong, X., \& Kirkpatrick, R. J. (1996). 29Si MAS NMR study of the structure of calcium silicate hydrate. Advanced Cement Based Materials, 3, 144-156. 
Constantinides, G., \& Ulm, F.-J. (2004). The effect of two types of $\mathrm{C}-\mathrm{S}-\mathrm{H}$ on the elasticity of cement-based materials: Results from nanoindentation and micromechanical modeling. Cement and Concrete Research, 34, 67-80.

Cullity, B. D. (1978). Elements of X-ray diffraction. Reading, MA: Addison-Wesley.

Duxson, P., Provis, J. L., Lukey, G. C., \& van Deventer, J. S. J. (2007). The role of inorganic polymer technology in the development of 'green concrete'. Cement and Concrete Research, 37, 1590-1597.

Eggert, J. H., Weck, G., Loubeyre, P., \& Mezouar, M. (2002). Quantitative structure factor and density measurements of high-pressure fluids in diamond anvil cells by X-ray diffraction: Argon and water. Physical Review B, 65, 174105

Gilbert, P. U. P. A., Metzler, R. A., Zhou, D., Scholl, A., Doran, A., Young, A., et al. (2008). Gradual ordering in red abalone nacre. Journal of the American Chemical Society, 130, 17519-17527.

Ha, J., Chae, S., Chou, K. W., Tyliszczak, T., \& Monteiro, P. J. M. (2011). Effect of polymers on the nanostructure and on the carbonation of calcium silicate hydrates: A scanning transmission X-ray microscopy study. Journal of Materials Science, 27, 1-14.

Hawthorne, F. C. (1988). Spectroscopic methods in mineralogy and geology. Washington, D.C.: Mineralogical Society of America.

Hong, X., Shen, G., Prakapenka, V. B., Rivers, M. L., \& Sutton, S. R. (2007). Density measurements of noncrystalline materials at high pressure with diamond anvil cell. Review of Scientific Instruments, 78, 103905-103906.

Howells, M., Kirz, J., Sayre, D., \& Schmahl, G. (1985). Soft-Xray microscopes. Physics Today, 38, 22-32.

Jackson, M. D., Vola, G., Všianský, D., Oleson, J. P., Scheetz, B. E., Brandon, C., \& Hohlfelder, R. L. (2012). Cement microstructures and durability in ancient roman seawater concretes. Historic Mortars, 7, 49-76.

John, D. B., Wenge, Y., Nobumichi, T., Jin-Seok, C., Jonathan, Z. T., Bennett, C. L., et al. (2003). X-ray microdiffraction study of growth modes and crystallographic tilts in oxide films on metal substrates. New York, NY: Nature Publishing Group.

Jones, M. R., Macphee, D. E., Chudek, J. A., Hunter, G., Lannegrand, R., Talero, R., et al. (2003). Studies using ${ }^{27} \mathrm{Al}$ MAS NMR of AFm and AFt phases and the formation of Friedel's salt. Cement and Concrete Research, 33, 177-182.

Juenger, M. C. G., Winnefeld, F., Provis, J. L., \& Ideker, J. H. (2011). Advances in alternative cementitious binders. Cement and Concrete Research, 41, 1232-1243.

Kilcoyne, D., Ade, H., Attwood, D., Hitchcock, A., McKean, P., Mitchell, G., Monteiro, P., Tyliszczak, T., \& Warwick, T. A new scanning transmission X-ray Microscope at the ALS for operation up to $2500 \mathrm{eV}$, In: AIP Conference Proceedings, 2010, p. 465.

Kilcoyne, A., Tyliszczak, T., Steele, W., Fakra, S., Hitchcock, P., Franck, K., et al. (2003). Interferometer-controlled scanning transmission X-ray microscopes at the advanced light source. Journal of Synchrotron Radiation, 10, $125-136$.
Koningsberger, D. C., \& Prins, R. (1987). X-ray absorption: Principles, applications, techniques of EXAFS. Wiley: SEXAFS and XANES.

Kunz, M., MacDowell, A. A., Caldwell, W. A., Cambie, D., Celestre, R. S., Domning, E. E., et al. (2005). A beamline for high-pressure studies at the advanced light source with a superconducting bending magnet as the source. Journal of Synchrotron Radiation, 12, 650-658.

Kunz, M., Tamura, N., Chen, K., MacDowell, A. A., Celestre, R. S., Church, M. M., et al. (2009). A dedicated superbend $\mathrm{X}$-ray microdiffraction beamline for materials, geo-, and environmental sciences at the advanced light source. Review of Scientific Instruments, 80, 035108-035110.

Langford, J. (1975). X-ray diffraction procedures for polycrystalline and amorphous materials by H. P. Klug and L. E. Alexander. Journal of Applied Crystallography, 8, 573-574.

Liu, Y., Wang, J., Hong, Y., Wang, Z., Zhang, K., Williams, P. A., et al. (2012). Extended depth of focus for transmission X-ray microscope. Optics Letters, 37, 3708-3710.

Mehta, P., \& Monteiro, P. (2006). Concrete-structure, material, and properties. Saddle River, NJ: Prantice Hall Inc.

Monteiro, P. J. M., Kirchheim, A. P., Chae, S., Fischer, P., MacDowell, A. A., Schaible, E., et al. (2009). Characterizing the nano and micro structure of concrete to improve its durability. Cement \& Concrete Composites, 31, $577-584$

Moon, J., Oh, J. E., Balonis, M., Glasser, F. P., Clark, S. M., \& Monteiro, P. J. M. (2011). Pressure induced reactions amongst calcium aluminate hydrate phases. Cement and Concrete Research, 41, 571-578.

Moon, J., Oh, J. E., Balonis, M., Glasser, F. P., Clark, S. M., \& Monteiro, P. J. M. (2012a). High pressure study of low compressibility tetracalcium aluminum carbonate hydrates $3 \mathrm{CaO} \cdot \mathrm{Al}_{2} \mathrm{O}_{3} \cdot \mathrm{CaCO}_{3} \cdot 11 \mathrm{H}_{2} \mathrm{O}$. Cement and Concrete Research, 42, 105-110.

Moon, J., Yoon, S., Wentzcovitch, R. M., Clark, S. M., \& Monteiro, P. J. M. (2012b). Elastic properties of tricalcium aluminate from high-pressure experiments and first-principles calculations. Journal of the American Ceramic Society, 95, 2972-2978.

Nyilas, R. D., Kobas, M., \& Spolenak, R. (2009). Synchrotron $\mathrm{X}$-ray microdiffraction reveals rotational plastic deformation mechanisms in polycrystalline thin films. Acta Materialia, 57, 3738-3753.

Oh, J. E., Clark, S. M., \& Monteiro, P. J. M. (2011). Does the Al substitution in $\mathrm{C}-\mathrm{S}-\mathrm{H}(\mathrm{I})$ change its mechanical property? Cement and Concrete Research, 41, 102-106.

Oh, J. E., Clark, S. M., Wenk, H.-R., \& Monteiro, P. J. M. (2012). Experimental determination of bulk modulus of $14 \AA$ tobermorite using high pressure synchrotron X-ray diffraction. Cement and Concrete Research, 42, 397-403.

Ohuchi, T., Kawazoe, T., Nishihara, Y., Nishiyama, N., \& Irifune, T. (2011). High pressure and temperature fabric transitions in olivine and variations in upper mantle seismic anisotropy. Earth and Planetary Science Letters, 304, $55-63$. 
Rehbein, S., Heim, S., Guttmann, P., Werner, S., \& Schneider, G. (2009). Ultrahigh-resolution soft-X-ray microscopy with zone plates in high orders of diffraction. Physical Review Letters, 103, 110801.

Robert, M. V. H., Winarski, P., Rose, V., Fuesz, P., Carbaugh, D., Benson, C., et al. (2012). A hard x-ray nanoprobe beamline for nanoscale microscopy. Journal of Synchrotron Radiation, 19, 1056-1060.

Sato, T., \& Funamori, N. (2008). High-pressure in situ density measurement of low- $Z$ noncrystalline materials with a diamond-anvil cell by an X-ray absorption method. Review of Scientific Instruments, 79, 073906-073911.

Schlachter, A. S. (1994). New Directions in research with thirdgeneration soft X-ray synchrotron radiation sources: Proceedings of the NATO advanced study institute on new directions in research with third-generation soft X-ray synchrotron radiation sources. Dordrecht, Netherlands: Kluwer academic publishers.

Schneider, G., Guttmann, P., Heim, S., Rehbein, S., Mueller, F., Nagashima, K., et al. (2010). Three-dimensional cellular ultrastructure resolved by X-ray microscopy. Nature Methods, 7, 985-987.

Schneider, G., Guttmann, P., Rehbein, S., Werner, S., \& Follath, R. (2012). Cryo X-ray microscope with flat sample geometry for correlative fluorescence and nanoscale tomographic imaging. Journal of Structural Biology, 177, 212-223.

Scrivener, K., Füllmann, T., Gallucci, E., Walenta, G., \& Bermejo, E. (2004). Quantitative study of Portland cement hydration by X-ray diffraction/Rietveld analysis and independent methods. Cement and Concrete Research, 34, $1541-1547$

Shen, G., Sata, N., Taberlet, N., Newville, M., Rivers, M. L., \& Sutton, S. R. (2002). Melting studies of indium:
Determination of the structure and density of melts at high pressures and high temperatures. Journal of Physics Condensed Matter, 14, 10533.

Speziale, S., Reichmann, H. J., Schilling, F. R., Wenk, H. R., \& Monteiro, P. J. M. (2008). Determination of the elastic constants of portlandite by Brillouin spectroscopy. Cement and Concrete Research, 38, 1148-1153.

Tamura, N., Kunz, M., Chen, K., Celestre, R. S., MacDowell, A. A., \& Warwick, T. (2009). A superbend X-ray microdiffraction beamline at the advanced light source. Materials Science and Engineering A, 524, 28-32.

Tamura, N., MacDowell, A. A., Spolenak, R., Valek, B. C., Bravman, J. C., Brown, W. L., et al. (2003). Scanning $\mathrm{X}$-ray microdiffraction with submicrometer white beam for strain/stress and orientation mapping in thin films. Journal of Synchrotron Radiation, 10, 137-143.

Webb, A. G. (2002). Introduction to Biomedical Imaging. New York, NY: Wiley.

Wenk, H.-R., Monteiro, P. J. M., Kunz, M., Chen, K., Tamura, N., Lutterotti, L., et al. (2009). Preferred orientation of ettringite in concrete fractures. Journal of Applied Crystallography, 42(3), 429-432.

Wieland, E., Dähn, R., Vespa, M., \& Lothenbach, B. (2010). Micro-spectroscopic investigation of $\mathrm{Al}$ and $\mathrm{S}$ speciation in hardened cement paste. Cement and Concrete Research, $40,885-891$.

Xiao, X., Liu, H., Wang, L., \& De Carlo, F. (2010). Density measurement of samples under high pressure using synchrotron micro tomography and diamond anvil cell techniques. Journal of Synchrotron Radiation, 17, 360-366.

Yarris, L. (2001). Ernest Orlando Lawrence: The man, his lab, his legacy, in: Science Beat (http://wwwlblgov/ScienceArticles/Archive/lawrence-legacyhtml), Lawrence Berkeley National Lab, Berkeley, CA. Accessed 1 Nov 2012. 